\title{
Limits on the flux of heavy compact objects from the the "Pi of the Sky" project
}

L W Piotrowski ${ }^{a, *}$ on behalf of the Pi of the Sky Collaboration

(a complete list of authors can be found at the end of the proceedings)

${ }^{a}$ Faculty of Physics, University of Warsaw - Warsaw, Poland

E-mail: publ@lwp.email

The existence of heavy compact objects has been suggested many times throughout the years. In terms of sizes, they would belong to the realms of nuclear or atomic physics, but in terms of masses could extend to the macroscopic world, reaching kilograms, tones or more. The most notable candidates are nuclearites - hypothetical lumps of strange quark matter, Q-Balls, magnetic monopoles or primordial black holes. These objects may have originated in the early universe or could be produced by some stellar phenomena. Especially in the first case, they could be a component of dark matter.

If they exist, it is likely that they reach our planet at high speeds and cross the atmosphere, leaving behind a trail of light in the air. We present results of a search for such objects in visual photographs of the sky taken by the "Pi of the Sky" experiment, exemplified with the most stringent limits on the flux of incoming nuclearites of the masses spanning between $100 \mathrm{~g}$ and $100 \mathrm{~kg}$.

$37^{\text {th }}$ International Cosmic Ray Conference (ICRC 2021)

July 12th - 23rd, 2021

Online - Berlin, Germany

\footnotetext{
*Presenter
} 


\section{Introduction}

The most extreme case of a heavy compact object that has ever been detected experimentally is a black hole - an object so heavy that it packs all its mass in a possibly infinitely small amount of space. The ones that have been observed so far are extremely heavy. Not much prevents, however, the existence of much lighter counterparts. They are just but one example of many types of heavy compact objects predicted by different branches of physics and astrophysics.

One other example is nuclearites - a name usually attached to heavy strangelets, hypothetical lumps of "strange quark matter" predicted by Witten[1], consisting of roughly equal numbers of up, down and strange quarks and being more stable than the ordinary matter consisting of only up and down quarks. De Rujula and Glashow predicted[2] that nuclearites, travelling with speeds of the order of $100 \mathrm{~km} / \mathrm{s}$ would collide elastically with atoms. Their energy loss mechanism would be similar to that of a meteor, however, their compact size would allow those heavier than $4 \times 10^{-14} \mathrm{~g}$ to penetrate the atmosphere, and those heavier than $0.1 \mathrm{~g}$ to pass through the Earth's diameter. In the process of traversing through a transparent medium such as air or water, they would create an expanding thermal shock-wave and thus convert part of their energy into visible light. The amount depends mainly on the density of the medium, and speed and radius of the object. The reasoning developed for nuclearites can be applied to different hypothetical compact objects that could interact with atoms in a similar manner.

For many of these candidates, including nuclearites, the light emitted in the atmosphere would create a light trail similar to that of meteors, but mostly in the lower atmosphere and reaching the ground. They would have a small loss of energy, no loss of mass and nearly constant speed, and thus produce a track with almost constant absolute brightness. In addition, Solar System meteors do not exceed speeds of $72 \mathrm{~km} / \mathrm{s}$, while in the scenarios of massive compact objects being of galactic or extragalactic origin, bound to the Galaxy as Dark Matter (DM), or coming from collisions or explosions of astrophysical objects, their speeds in most cases would be at least a few times higher. Despite the differences, they would be observable by star-gazing with on-ground telescopes. Those monitoring large parts of the sky, like the "Pi of the Sky" experiment, would be the most likely to detect them, and an attempt to do so has been described in [3].

\section{The "Pi of the Sky" experiment}

The "Pi of the Sky" experiment[4] was a system of wide field of view robotic telescopes designed to search for variable astrophysical phenomena. The design of the apparatus allowed to monitor a large fraction of the sky with a range of $12^{m}-13^{m_{1}}$ (these values are not used for obtaining the results presented in this manuscript, as they are too general) and time resolution of the order of 10 seconds. The main goal of the project was to search for optical counterparts of Gamma Ray Bursts (GRBs) during or even before gamma-ray emission[5, 6].

\footnotetext{
${ }^{1}$ Magnitude ${ }^{\mathrm{m}}$ stands for an apparent brightness of an astronomical object in relation to an object of reference: $m=-2.5 \log \frac{I}{I_{r}}+m_{r}$, where $m_{r}$ is the magnitude of the object of reference, $I_{r}$ its observed radiation flux and $I$ is the observed radiation flux of the discussed object. This unit has been normalized according to the Ptolemy scale, where the brightest stars seen with the naked eye had a brightness of $1^{\mathrm{m}}$ and the darkest of $6^{\mathrm{m}}$. The Sun has a brightness of $-26^{\mathrm{m}}$, and Vega $0^{\mathrm{m}}$.
} 
The experiment was equipped with custom-designed cameras and Canon lenses with focal length $\mathrm{f}=85 \mathrm{~mm}, \mathrm{f} / \mathrm{d}=1.2$ ( $\mathrm{d}$ standing for the diameter of the entrance pupil), each camera covering $\sim 20^{\circ} \times 20^{\circ}$ of the sky with roughly 4 million pixels. The full system consisted of 16 cameras placed on equatorial mounts (4 cameras per mount), covering almost 2 sr of the sky, working since 2013 in the INTA El Arenosillo test centre in Mazagón near Huelva, Spain. Before that, a prototype consisting of 2 cameras working in coincidence and observing the same field of view had been working at Las Campanas Observatory in Chile since June 2004 until the end of 2009, and later in San Pedro de Atacama in Chile. The project stopped gathering data in 2017.

\section{Search for compact heavy objects}

The $10 \mathrm{~s}$ exposure time of the "Pi of the Sky" cameras is not optimised for fast-moving heavy compact objects. The time spent in any of the camera's pixels is very short compared to the whole exposure and decreases the signal to noise ratio, and thus limits the sensitivity to low mass, dim events. However, the experiment was monitoring a very large part of the atmosphere and gathered a significant amount of data, useful for looking for small fluxes.

Using a custom track search algorithm, we have analysed our archived raw data: 337674 frames with $10 \mathrm{~s}$ exposure and 34004 stacked frames, consisting of $20 \times 10 \mathrm{~s}$ exposures from many locations on the sky. Among those 185258 single and 22237 stacked frames were left after quality cuts and a requirement that the centre of the field of view is pointing more than $20^{\circ}$ above the horizon. This adds up to $1766.05 \mathrm{~h}$ of clear observation for a single camera equivalent.

After performing track detection and initial removal of spurious events (clouds, cosmic rays, etc.) on the frames, 35870 tracks (corresponding to the $1766.05 \mathrm{~h}$ residual observation

time) passed on to the next analysis stage. Among those, 33257 were automatically classified as meteors or satellites and 2613 underwent manual inspection.

The manual inspection consisted of analysing intensity profiles and track images. The main challenge is to distinguish between nuclearites and meteors/satellites. The change in intensity of a nuclearite signal comes only from the changing distance to the detector and is thus expected to be small, very smooth and slow. Therefore all events with rapid changes in brightness have been classified as meteors or satellites. The inspection left 29 candidates for nuclearites.

The 29 remaining tracks were compared with the NORAD satellite database for the corresponding nights and 9 events were identified as satellites, which left us with 20 candidates for nuclearites. However, the NORAD satellite database is far from complete, and it is quite likely that many other of those events are satellites.

None of the 20 events is an obvious candidate for a nuclearite track, which should be very long, with an almost constant and preferably strong signal. The longest one is about 1055 pixels, exiting the frame (fig. 1). One could speculate about multiple trends in the presented intensity profile which should not exist for a real nuclearite, however definite exclusion of this event is hard.

\section{Limits on the flux of heavy compact objects}

To estimate the limits on the flux of heavy compact objects in the "Pi of the Sky" experiment, we need to establish the maximal achievable flux for the selection of object masses and multiply 

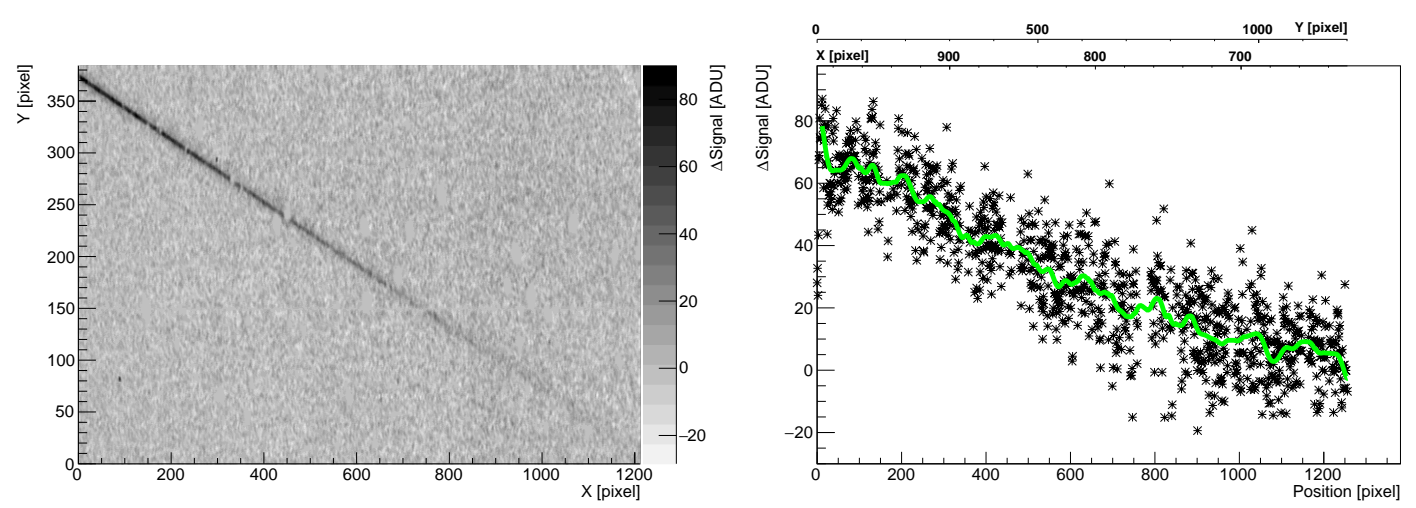

Figure 1: The longest of the remaining nuclearite candidates. Left: the track on the frame after subtraction of the previous frame and Gaussian smoothing. Right: the intensity profile of the event, with the curve showing the Gaussian smoothing of the measurement points.

it by the efficiency of detection and separation from other types of tracks in the atmosphere. For this purpose we have simulated nuclearites crossing our field of view with brightness in stellar magnitude units:

$$
\mathcal{M}=10.8-1.67 \log _{10}(m / 1 \mu g)+5 \log _{10}(d / 10 \mathrm{~km})
$$

where $m$ is the mass and $d$ is the distance to the telescope. The maximal altitude at which nuclearites effectively generate light is:

$$
h_{\text {max }}=2.7 \mathrm{~km} \ln \left(\mathrm{m} / 1.2 \times 10^{-5} \mathrm{~g}\right)
$$

both equations following the calculations of De Rujula and Glashow[2]. Next, we applied our detectors' parameters such as exposure, PSF, pointing zenith angle, atmospheric extinction, etc. Using this procedure we have determined the detector's effective surface (including detection efficiency) and thus calculated limits for an isotropic flux and directional flux caused by Earth's movement in a "sea" of a static halo of nuclearites.

\subsection{Isotropic limiting flux}

The isotropic flux of heavy compact objects could come from extragalactic sources such as GRBs or Galactic sources with isotropic distribution around the Earth. It is also used as an approximation of a flux of Dark Matter objects by De Rujula and Glashow following the isothermal sphere assumption of the Dark Matter's Standard Halo Model (SHM).

We have simulated a random isotropic flux of nuclearites crossing the volume of the atmosphere observed and then supplied the results to the detection algorithm, to estimate our detector's effective area $S_{E}^{I s o}(m)$, which is approximated by the surface of the field of view pyramid of a camera and depends on nuclearite mass $m$, sky conditions, and the detector's pointing zenith angle. This results in the following formula for the $90 \% \mathrm{CL}$ (confidence level) limit on the isotropic flux:

$$
\Phi(m)=\frac{2.3}{S_{E}^{I s o}(m) \cdot t_{e} \cdot 2 \pi}
$$




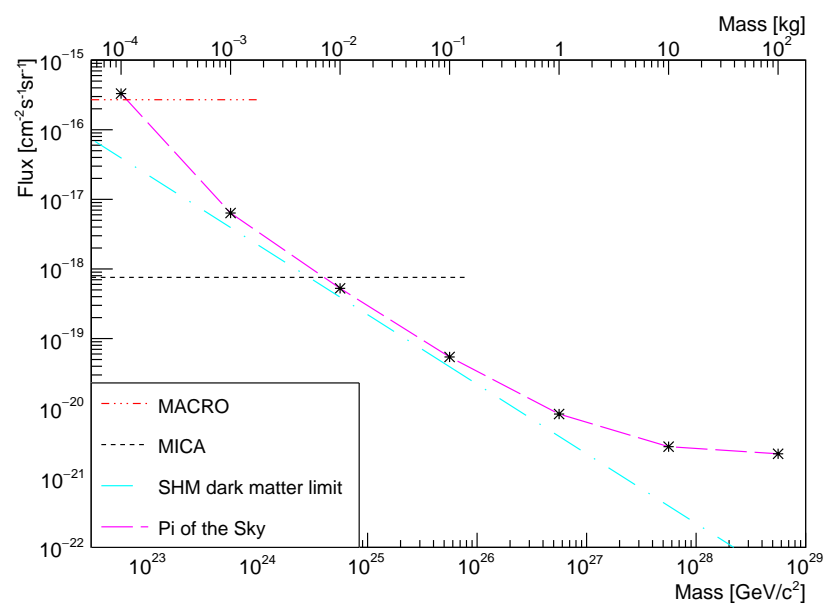

Figure 2: $90 \%$ CL limit for the isotropic flux of nuclearites of specific mass by the "Pi of the Sky" project on top of the limits given by MACRO and a mica minerals analysis. We show also the constraint in the SHM dark matter scenario, assuming its isotropic flux on Earth.

where $t_{e}$ stands for the exposure time (10 s for single and $200 \mathrm{~s}$ for stacked frames) and $2 \pi$ comes from the fact that we do not take into account nuclearites coming from below the detector. Fig. 2 shows the obtained flux limit on top of the limits given by MICA [7] and MACRO[8]. It is important to mention that SLIM has given a limit of $\sim 10^{-15} \mathrm{~cm}^{-2} \mathrm{~s}^{-1} \mathrm{sr}^{-1}$ for nuclearites with mass below $10^{21} \mathrm{GeV} / \mathrm{c}^{2}$ [9], and ANTARES a limit of $\sim 10^{-17} \mathrm{~cm}^{-2} \mathrm{~s}^{-1} \mathrm{sr}^{-1}$ for nuclearites with mass below $10^{17} \mathrm{GeV} / \mathrm{c}^{2}[10]$.

\subsection{Directional limiting flux}

Here we consider a case where nuclearites are static in the Galaxy and bombard the Earth due to its movement through the Milky Way along with the Solar System. This can be used as a basis for calculations involving more complicated assumptions about distributions and velocities of compact heavy objects. In this case, the flux will not be isotropic but aligned along the telescope's velocity vector. The $90 \% \mathrm{CL}$ limit on the directional flux is given by:

$$
\Phi(m)=\frac{2.3}{S_{E}(m) \cdot t_{e}}
$$

losing the $2 \pi$ factor from the isotropic flux. The effective surface $S_{E}(m)$ includes the field of view pyramid's cross-section perpendicular to the flux direction for a specific frame. The flux limit is drawn in fig. 3. Only 142772 single and 7984 stacked frames where the flux was coming from above the horizon were taken into account.

\section{Discussion of the results}

Presented results can be applied to heavy compact objects, assuming they enter the atmosphere with a speed of the order of hundreds of kilometres per second and interact with the atoms elastically or semi-elastically. The assumption is based on the well-known behaviour of meteors and orbital 


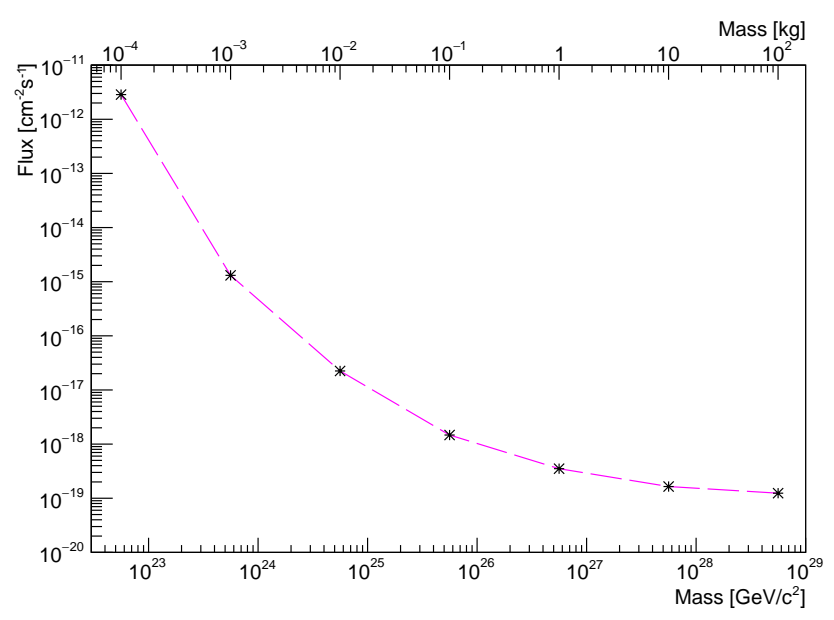

Figure 3: $90 \%$ CL limit for the directional flux of nuclearites of specific mass by the "Pi of the Sky" project

objects during orbital re-entry. The mass-scale, selected for nuclearites, assumes that the crosssection is purely geometrical. The results can be used for different objects after the mass-scale is adjusted taking into account their cross-section or different light emission mechanisms.

The flux limit value is determined mainly by the experiment's field of view, exposure time and number of frames analysed. The estimation of the detector's efficiency in detecting objects of specific mass plays a secondary role in the presented mass-region, and possible deviations from estimated values due to simulation uncertainties would hardly be visible on the logarithmic flux scale. Only downward going objects were taken into account. The limits could be simply divided by 2 for $1 \mathrm{~kg}$ and heavier nuclearites, if upward going objects were to be considered, as in this mass range traversing the Earth causes almost no speed loss. The selection efficiency could be improved with a detailed analysis of the brightness of the selected events and comparing them to specific objects masses.

The presented mass range could be extended to higher masses until tracks become so bright as to saturate the detector. Assessing when this happens is out of the scope of this paper, but it is clear from the presented curve, that the limit would not change significantly (strengthening slightly due to the increasing maximal altitude at which nuclearites effectively generate light). With enough time and computing power invested into further simulations, we could extend the limits to lower masses, but due to extremely small efficiencies, the possibility of detection would rely on a very small chance that a heavy compact object passes very close to the detector.

\subsection{Constraints on Dark Matter}

De Rujula and Glashow estimated an upper bound on the flux of nuclearites as an isotropic flux of Dark Matter on Earth given by $F=7.8 \cdot(1 \mathrm{~g} / \mathrm{m})$ (where $\mathrm{m}$ is the mass of a nuclearite) based on the local DM density of the SHM. Our isotropic limits are weaker than this bound, with the closest point being $~ 60 \%$ above (unless uncertainties of the SHM are considered). Fine-tuning our analysis, such as taking into account upward going nuclearites, would put us on the other side of the upper bound line. However, even in this case, we would not attempt to put constraints on 
the SHM. One has to realise that the upper bound assumes a flux of the same mass objects. This could be true for an elementary particle, but not for a bag of quarks with an unknown, but probably mostly continuous spectrum. Also, it is important to mention that the local dark matter density

is uncertain within a factor of a few, and the SHM approximation

may prove overly simplistic. Finally, the assumption of an isotropic flux of DM composites on the Earth requires the planet to be stationary in the Galaxy. Therefore we find our limits, especially the directional limit as a good basis for limit calculations for specific compact objects and their expected flux distributions, and the isotropic limits for non-DM origin scenarios.

It is worth noting that for objects with a higher interaction cross-section, the limit curve shifts towards lower masses. For example, for magnetised nuclearites[11], the shift is about 9 orders of magnitudes, allowing for constraining the SHM.

However, as shown in [12] and [13], the light production mechanism for nuclearites could be different than the one proposed by de Rujula and Glashow, and result in magnitudes lower by many orders of magnitude. In such a case, the presented limits for given masses would be significantly worse.

\section{Conclusions}

We have shown flux limits on compact heavy objects, in the mass scale range considered for nuclearites. These are the most stringent limits up to date in the $100 \mathrm{~g}-100 \mathrm{~kg}$ range according to the authors' knowledge. The limits can be extended to much higher masses until the object becomes too bright to be recognised as a track. The isotropic limit can be used mainly under the assumption of extragalactic sources such as short GRBs, which, in case they are colliding strange stars could eject nuclearites, which in turn, if beamed like electromagnetic radiation, could perhaps supply a significant flux on the Earth. The directional flux can serve as a basis for calculations involving specific scenarios of spatial and velocity distributions of objects and could be useful in investigations of dark matter hypotheses, especially if the mass scale is shifted towards lower masses, which would be the case for objects with higher cross-section, such as magnetised nuclearites. Finally, these limits may result in constraints on a growing number of astrophysical scenarios, ranging from early Universe evolution to cosmic cataclysms, where the production of heavy compact objects is expected.

\section{Acknowledgments}

K.M. has been supported by the Polish National Science Centre (UMO-2018/30/E/ST9/00082).

\section{References}

[1] E. Witten, Cosmic separation of phases, Physical Review D 30 (1984) 272.

[2] A. De Rújula and S.L. Glashow, Nuclearites-a novel form of cosmic radiation, Nature 312 (1984) 734. 
[3] L.W. Piotrowski, K. Małek, L. Mankiewicz, M. Sokołowski, G. Wrochna, A. Zadrożny et al., Limits on the flux of nuclearites and other heavy compact objects from the pi of the sky project, Phys. Rev. Lett. 125 (2020) 091101.

[4] A. Burd et al., Pi of the Sky-all-sky, real-time search for fast optical transients, New Astronomy 10 (2005) 409.

[5] J.L. Racusin, S.V. Karpov, M. Sokolowski, J. Granot, X. Wu, V. Pal'Shin et al., Broadband observations of the naked-eye $\gamma$-ray burst GRB 080319B, Nature 455 (2008) 183.

[6] R. Opiela et al., Pi of the Sky observation of grb160625b, in Photonics Applications in Astronomy, Communications, Industry, and High Energy Physics Experiments 2017, vol. 10445, p. 104454C, International Society for Optics and Photonics, 2017.

[7] P.B. Price, Limits on contribution of cosmic nuclearites to galactic dark matter, Physical Review D 38 (1988) 3813.

[8] MACRO collaboration, Nuclearite search with the MACRO detector at Gran Sasso, The European Physical Journal C-Particles and Fields 13 (2000) 453.

[9] S. Cecchini, M. Cozzi, D. Di Ferdinando, M. Errico, F. Fabbri, G. Giacomelli et al., Results of the search for strange quark matter and Q-balls with the SLIM Experiment, The European Physical Journal C 57 (2008) 525.

[10] ANTARES collaboration, Search for massive exotic particles with the ANTARES neutrino telescope, in Journal of Physics: Conference Series, vol. 409, p. 012135, IOP Publishing, 2013.

[11] J.P. VanDevender, A.P. VanDevender, T. Sloan, C. Swaim, P. Wilson, R.G. Schmitt et al., Detection of magnetized quark-nuggets, a candidate for dark matter, Scientific reports 7 (2017) 8758.

[12] J.S. Sidhu and G. Starkman, Macroscopic dark matter constraints from bolide camera networks, Physical Review D 100 (2019) 123008.

[13] L.A. Anchordoqui et al., Prospects for macroscopic dark matter detection at space-based and suborbital experiments, arXiv preprint arXiv:2104.05131 (2021) . 


\section{Full Authors List: Pi of the Sky Collaboration}

Katarzyna Małek ${ }^{12}$, Lech Mankiewicz ${ }^{3}$, Lech Wiktor Piotrowski ${ }^{4}$, Marcin Sokołowski $^{5}$, Grzegorz Wrochna ${ }^{6}$, Adam Zadrożny ${ }^{6}$, Aleksander Filip Żarnecki ${ }^{4}$

${ }^{1}$ National Centre for Nuclear Research, ul. Pasteura 7, 02-093 Warsaw, Poland ${ }^{2}$ Aix Marseille Univ. CNRS, CNES, LAM, Marseille, France ${ }^{3}$ Centre for Theoretical Physics, Polish Academy of Science, Warsaw, Poland ${ }^{4}$ Faculty of Physics, University of Warsaw, Warsaw, Poland ${ }^{5}$ International Centre for Radio Astronomy Research, Curtin University, Bentley, WA 6102, Australia ${ }^{6}$ National Centre for Nuclear Research, ul. Pasteura 7, 02-093 Warsaw, Poland 